Chapter 24

\title{
Periprosthetic Infection \\ Following Total Knee Arthroplasty
}

\author{
Michael Soudry, Arnan Greental, \\ Gabriel Nierenberg, Mazen Falah and \\ Nahum Rosenberg \\ Additional information is available at the end of the chapter \\ http://dx.doi.org/10.5772/53250
}

\section{Introduction}

One of the most devastating complications of prosthetic knee arthroplasty is a periprosthetic infection. This complication occurs in $1-2 \%$ of knee arthroplasties $[1,2]$ and can exceed $4 \%$ in immunocompromized individuals [3] and 7\% after revision surgery [4]. Prosthetic infection leads to loosening of the implant, [5,6]. In this circumstances revision surgery is required. Because of the diversity of the clinical presentation, i.e. early, intermediate or late infection [1], different surgical methods to treat infected knee prostheses were developed [5,6]. Several treatment methods became well accepted but others are still controversial. In the present review we intend to describe mainly the diagnostic tools for detection of infection and commonly used treatment methods in failed total knee arthroplasty due to infection, with special emphasis on the surgical techniques. Additionally we will describe some trends for the future improvement of the treatment modalities.

\section{Pathology and microbiology}

The main infecting pathogens, around $50 \%$, of knee prostheses, are the different strains of Staphylococci, e.g. coagulase negative Staphylococci cause around $27 \%$ of knee prostheses infections and Staphylococcus aureus is responsible for $23 \%$ of infections, according to pooled data from nine different studies [7]. Most of the clinically significant infections are caused by biofilm producing microorganisms. The role of biofilms in pathogenesis of periprosthetic infection is the masking of the pathogens from bodily immune response and antibiotic access. Biofilm is a biological 
structure containing bacteria in a planktonic form imbedded in extracellular matrix made of different polysaccharide molecules, proteins and extracellular DNA (Figure 1). Biofilm generation goes through four consecutive steps: adherence of the pathogens to the surface of prosthesis, accumulation of the biofilm components, maturation of the biofilm and finally its detachment and spread of the microorganisms [8]. The ability of the microorganism to produce masking biofilm defines its virulence in prosthetic infection. Commensal bacteria, such as coagulase negative Staphylococci are more frequent in immediate and early prosthetic infections, when spread from the surgical wound edges and in late low grade infections. In late infections by hematogeous spread the Staphylococcus aureus is the most important causative factor [9].

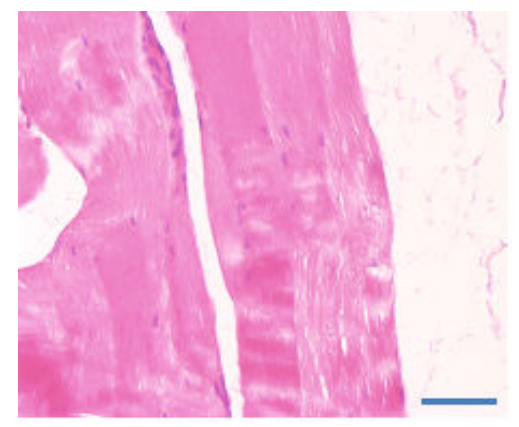

Figure 1. Microscopic image (H\&E staining, scale 100 $\mu$ ) of biofilm found the edge of retrieved tibial component of infected knee prosthesis. Amorphous fibrin-like substance, mostly acellular, is evident

\section{Timing of occurence}

Infections associated with prosthetic joints are classified according to time at detection as: early (develop less than 3 months after surgery), delayed [ 3 to 24 months after surgery) or late (more than 24 months after surgery) [10]. Clinical manifestations are in relation with timing [11]. In early cases clinical manifestations are joint pain, effusion, erythema and warmth of the joint. In delayed cases there are subtle signs such as implant loosening, persistent joint pain. Infection is generally provoked by less virulent microorganism. Late are acquired during hematogenous seeding. In a study of infection with THA during a 16 years period, $29 \%$ of cases were early infections, $41 \%$ delayed and 30\% late infections [12].

\section{Diagnosis}

Accurate and early diagnosis is the first step in effectively managing patients with prosthetic joint infection. Clinical history, physical examination, laboratory data and imaging studies are all taken into consideration. In addition to cultures, the most commonly used laboratory tests include serum inflammatory markers and synovial fluid cytology. 
Plain films: The appearance of rapidly progressive radiolucent lines surrounding an implant may be present during an infection. The resorption of subchondral bone and patchy osteoporosis are strong elements of suspicion (Figure 2).

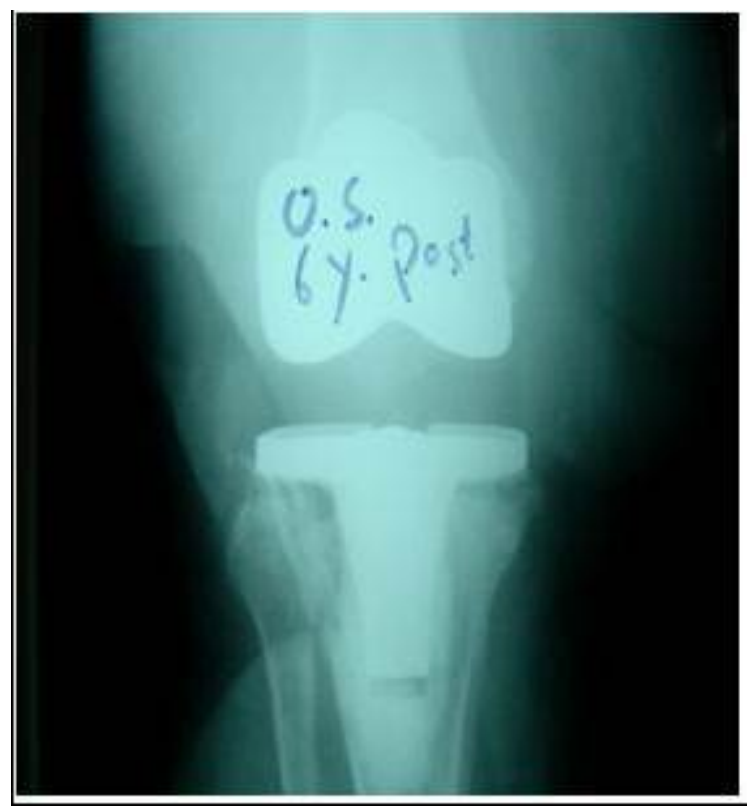

Figure 2. Radiograph of right knee (anterior-posterior view) showing radiolucency under the tibial component indicating periprosthetic infection.

Bone scan: bone scan can help confirm a diagnosis. However its high cost and its inability to provide acceptable levels of sensitivity and specificity have restricted its use. Although bone scintigraphy with technetium $99 \mathrm{~m}$ - labeled methylene diphosphonate has a high sensitivity, it lacks specificity for infection [13]. A technetium bone scan remains positive more than one year after implantation because of increased periprosthetic bone remodeling. Bone scan alone without labeling of the white cell has been found to have no role in diagnosing prosthetic joint infection. However, the use of indium 111 labeled leucocyte is time consuming and requires specialized labelling facility [14].

Laboratory tests: There is no evidence supporting the role of WBC and/or white cell blood differential in diagnosis of presence or absence of infection. ESR and CRP are valuable markers for both diagnosing and monitoring periprosthetic infection. After surgery the C Reactive protein level is elevated and return to normal within weeks. Serial postoperative measurements are more informative than single values [15].

Elevated serum interleukin-6 level correlated positively with the presence of periprosthetic infection in patients undergoing a reoperation at the site of a total hip or knee arthroplasty. 
In a prospective, case-control study of 58 patients undergoing revision surgery of total hip and knee arthroplasties, serum Interleukin- 6 values $>10 \mathrm{pg} / \mathrm{mL}$ was reported to have a sensitivity of $100 \%$, specificity of $95 \%$, positive predictive value of $89 \%$, negative predictive value of $100 \%$ and accuracy of $97 \%$ [16].

Knee aspirate cell count and differential: Synovial fluid cell count and differential is a very useful diagnostic test. Antibiotics should be suspended, if possible, for 10 to 14 days before carrying out the aspiration. Traumatic aspirations will result in falsely elevated leukocyte counts.

Polymerase chain reaction (PCR): This method is used to detect and amplify the presence of bacterial DNA. It is thought to be a quick method since it is not affected by whether the patient takes antibiotics or not. However, a high percentage of false negative test results has been detected [17]. Therefore currently this technique can be used as a complementary diagnostic tool to the methods described above.

Sonication: Organisms associated with prosthetic-joint infection are found attached to the prosthesis, where they often form biofilms. This suggests that obtaining a sample from the implant might improve the diagnosis of prosthetic joint infection by unmasking adherent bacteria from explanted prosthesis by sonication. It was found that culture of samples obtained by sonication of prostheses were more sensitive than conventional periprosthetic-tissue culture for the microbiologic diagnosis of prosthetic joint infection, especially in patients who had received antimicrobial therapy within 14 days before surgery [18].

Intraoperative Frozen Section: The analysis of frozen histological sections is a valuable tool for diagnosing infection. It most often used to assist decision-making in cases with equivocal serum inflammatory makers and aspirate cytology. The cutoff value of $>5$ neutrophils per high power field at a magnification of 400 is most commonly used for the diagnosis of infection. The sensitivity is more than 80 percent and a specificity of of more than 80 percent [19].

Intraoperative Gram Stain: This modality is unreliable (sensitivity $=27 \%$ ) and should not be used routinely. The AAOS guideline recommends against the use of intraoperative gram stain to rule out periprosthetic infection [20].

\section{Management of total knee arthroplasty infection}

There are several options when it comes to managing an infected TKA. But before we select any of these, we must take into consideration a series of factors. These factors include the amount of time elapsed from infection, host-related factors, condition of the soft tissues, condition of the implant, virulence of microorganism present and its degree of sensitivity and, last but not least, the patient's expectations and functional needs.

Planning for any one option requires having detailed clinical records, cultures, $\mathrm{x}$-rays and information of previously received treatment. It is important to identify high-risk patients, i.e. those receiving immunosuppressive treatment or suffering from malnutrition or systemic disease, trying to improve their general condition as much as possible before surgery. 
Physical examination should provide information about the patient's neurovascular situation, articular mobility, the condition of their extensor mechanism and their soft tissues as well as about any previous incisions or the need of skin coverage by a plastic surgeon. Preoperative planning is important. The final goal of treatment is to eradicate infection, ease the pain and preserve the limb's function.

\subsection{Antibiotic suppressive therapy}

Efficiency of infection eradication with antibiotic therapy only is limited mainly due to the presence of foreign bodies, as implant and acrylic cement, and bacterial biofilm, therefore its use should be restricted to specific circumstances [21,22].

Indications for this type of treatment are as follows: 1) High operative risk due to medical co-morbidities; 2) Presence of low-virulence micro-organisms susceptible oral antibiotics that can be tolerated by the patient; 3) Mechanically stable prosthesis.

Antibiotic treatment should follow 3 basic principles: 1) Use of antibiotics of proven intracellular efficacy such as rifampicin, and new anti-staphylococcal agents. 2) Antibiotics should be combined, using a minimum of two to enhance the possibility of therapeutic success. 3) Long-standing administration, i.e. treatment should last a minimum of 6 months.

The use of new antibiotics could improve results for resistant bacteria. The oxazolidinone linezolid is a new wide-spectrum antibiotic with very attractive pharmacokinetic and activity profiles. It is an antibiotic that acts against methicillin-resistant staphylococci and vancomycin-resistant enterococci.

\subsection{Surgical treatment}

In early infection debridement and irrigation, without removing the implant, are usually chosen for surgical treatment.

The approach is through the previous surgical wound. Following division the subcutaneous tissue the knee is aspirated again.

Beforehand, the surgeon should carefully evaluate the knee radiographs for any sign of loosening, slight change in the components position, heterotrophic bone formation. All these may indicate chronic situation. Following the surgical exposure the stability of the implant should be evaluated. If reactive tissue found to sprout at the edge of the implant, it also might indicate on chronicity of the infection.[23, 24, 25]

Extensive debridement should be performed followed with vigorous irrigation. The debrided tissue is sent for cultures and pathology while the implant preserved. When no reactive tissue left, last survey should include the gutters, the patellar tracking and the back fold of the knee.

Closure of the knee might need multi layers sutures, using non absorbable materials over heavy drain, which could be left in place for several days to the discharge to stop. Most surgeons allow regular rehabilitation and long term IV antibiotics. Some surgeons leave antibiotic beads and perform recurrent debridement prior to knee rehabilitation [23] 


\subsection{Delayed or late infections}

With delayed or late infection the orthopedic surgeon might face various clinical uncertainties with regard of decision making. The following are the most common clinical situations that are usually encountered:

Suppurating knee with positive cultures

Clinically infected knee with positive laboratory data but negative cultures

Clinically suspected infected knee without support of laboratory data

Clinically not infected knee with positive cultures

\subsubsection{Suppurating knee with positive cultures}

Identification of the infective germ prior to surgery allows preparation of appropriate antibiotics use within the operation. In some centers single stage revision preferred in cases of low virulence germ, effective antibiotics available both for embedding in the cement and the parenteral line.

Most surgeons favor a two-stage revision instead of a one-stage procedure [26, 27]

\subsubsection{The two stage procedure}

The two-stage procedure is indicated particularly to treat overt infections with an active discharge and virulent organism on culture such as Staphylococus aureus and mainly in methicillin-resistant staphylococcus (MRSA). Removal of the implant is done in first stage and implantation of the new prosthesis is performed later and delayed for a variable period of time until all parameters of inflammation disappear (Figure 3).

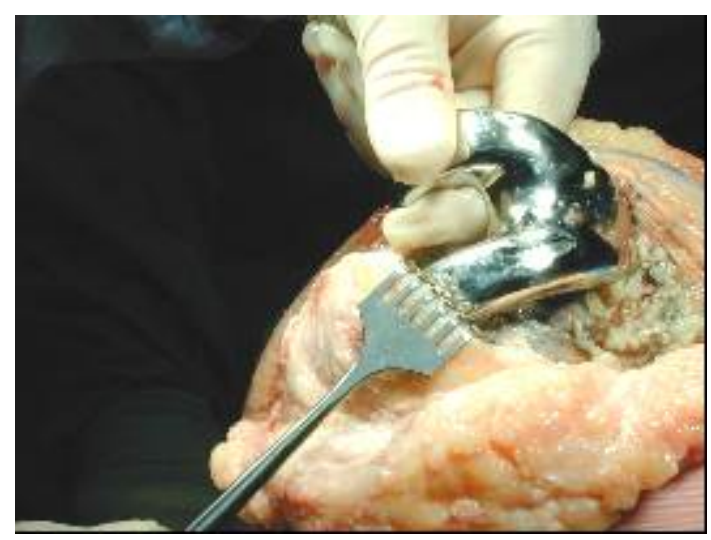

Figure 3. Intraoperative image of the grossly infected knee prosthesis before retrieval 
The use of tourniquet without Esmarch bandage is advisable. Careful marking of the scars allows excision of the scars with old suture material. The arthrotomy should follow the original cut with extended lengths if necessary. Careful dissection is utilized in order to protect the vulnerable subcutaneous flaps. If open sinuses exist they should be debrided through the track. Pus and soft tissue are sent to culture with long incubation [28]. Extensive meticulous debridement is performed to the level of natural tissue, removing all synovial necrotic and non viable tissue. [27,29]. All prosthetic components and acrylic cement are removed.

After the implants are exposed from all soft tissue, the anterior surface of the femoral component is gently released as with Gigli saw, the distal part of it is detached by thin osteotome and gentle mallet percussions saving the bone stock, without leaning on the soft infected bone. Following removal of the femoral component the undersurface of the tibial tray is released with a saw and osteotomes which are inserted medially and laterally. Then hammering of the tray away from the tibia is performed. Meticulous removal of all pieces of cement is a must and,although can be technically demanding, should be accomplished. Thorough debridement is performed again with excision and removal of all remnants of infected tissue. Then the dressing is changed and the knee is draped again. Irrigation should follow with 3 to 4 liters of saline. Five minutes of betadine soaking of the wound should be followed by insertion of antibiotic-impregnated spacers.

A cement spacer impregnated with eluting antimicrobial drugs, according the sensitivity of the infective microorganism, is then interposed between distal femur and proximal tibia. This keeps the limb at its correct length and allows partial joint mobility Non-articulating, or articulated, spacers, can be used according to preference. Few spacer types are used: antibiotic cemented beads, antibiotic cement block, articulating spacer etc [30, 31, 32] (Figure 4).
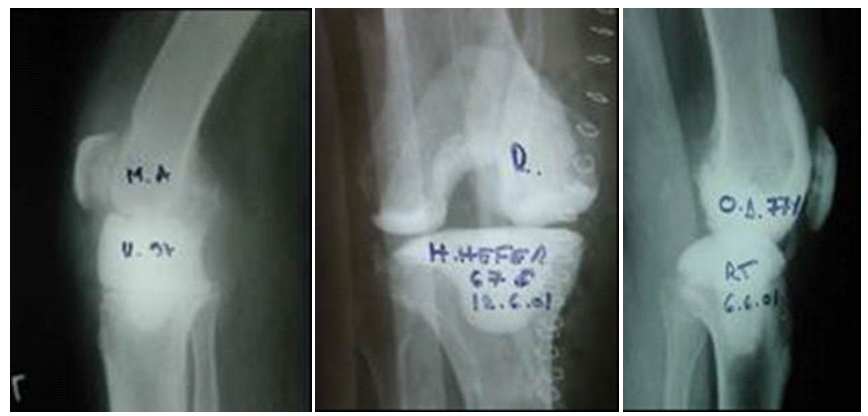

Figure 4. Knee radiographs showing different types of cement spacers

The non articulated spacer is a fixed one, with inherited stability that allows post op full weight bearing but no knee movement. Sometimes an intramedullary nail (abut $30-36 \mathrm{~cm}$ long) used to bridge the knee with cement for the enhanced stability. Care should be taken to prevent thermal injuries by the inserted cement at its' extension under the patella. 
A divided or articulated spacer contains two parts: one piece should be attached to the proximal tibia and the other to the distal femur. The articulating cement spacer allows the patient to bend his knee, to exercise for range of movement, thus preventing joint contractures and keeping the extensor mechanism integrity.

Adequate hemostasis should follow tourniquet release, irrigation and closure of the wound over a large bore drain.

Antibiotics are administered intravenously according to microbial sensitivity for an average of six weeks. The interval can vary between the two stages, e.g. between six weeks to three months, when the clinical condition is settled. During this period, the clinical recovery is carefully evaluated by the laboratory tests for infection control (ESR and CRP). If there is no clear evidence of clinical improvement, re-arthrotomy and debridement should be considered.

The second stage requires re-arthrotomy through the old scar, tissue is sent for cultures, for pathologic examination, including high power field microscopic examination. The cement spacer is removed; the surgeon should patiently repeat the meticulous debridement. Intense irrigation and change of knee dressing followed by bone preparation and revision implant cementing are performed. A constrained rotating knee prosthesis is generally the most suitable implant particularly in cases of bone loss.

\subsubsection{The one stage procedure}

The use of a single-stage revision is advocated by some in certain patients with known causative organism, when no discharging sinuses are present, the patient is not immunocompromized, and there is no radiological evidence of component loosening or osteitis.

This type of revision is considered when pathogen germ has been definitely identified with appropriate sensitive antibiotic. The cement should contain suitable antibiotics according the sensitivity of the infective pathogen, if it is known; antimicrobial treatment is given 2-3 weeks before prosthesis exchange.

Technically one stage revision procedure includes removal of all foreign material, implant components and cement, thorough the same steps of meticulous debridement, as stated above, and re-implantation of a new prosthesis at the same surgical session.

\subsubsection{Clinically infected knee with positive laboratory data but negative cultures}

Clinical infection with negative cultures is not rare. A patient may present painful and swollen knee, with synovitis and intraarticular fluid, elevated ESR and CRP, with positive leukocyte bone scan, while aspirated fluid reveal negative cultures. In such a case the aspiration should be repeated, and microbiological studies for rare microorganisms, including PCR should be performed.

The clinical suspicion mandates the type of surgery: The surgical process should be identical to $1^{\text {st }}$ stage revision with extensive debridement and removal of the implant. Multiple bone and soft tissue cultures and pathology should be obtained intraoperatively. Sonication of the prosthesis might be indicated. The cement spacer should contain antibiotics relevant against 
the common bacteria. Post operative intravenous antibiotics should be administered for six weeks. The $2^{\text {nd }}$ stage is similar to those performed for the positive culture group.

The one-stage type of revision can also be considered in presence of low grade clinical expression, such as long relentless pain, local heat, tenderness and slow rehabilitation milestones, negative preoperative aspiration cultures and intraoperative gram stains, as well as frozen section demonstrating less than 5 polymorphonuclears per high power field. Aged patients with positive cultures for low virulence strains, such as Staphylococcus epidermidis and Streptococcus type A, are sometimes allocated for revision in a single stage.

\subsubsection{Clinically suspected infected knee without support of laboratory data}

This group of patients is presented with swollen painful knee, sometimes with synovitis and loosening. Usually not long from the primary surgery, with no sign of polyethylene wear, normal laboratory tests as CRP or ESR, normal blood leukocytes count and negative leukocytes bone scan. Knee aspiration could reveal not clear fluid but with negative culture. In such circumstances repeated aspiration performed and the workup should be extended for material allergy such as nickel and chrome. If the clinical suspicion for infection is significant the surgeon might take steps as for fully infected case, performing two or single stage revision. The decision making in these circumstances lacks a high level of evidence support.

\subsubsection{Clinically not infected knee with positive cultures}

Bone and soft tissue cultures are part of all knee revision as well as routine sonication of the retrieved implants. Sometimes a positive culture might be discovered in routine, not infected, with normal blood tests, negative leukocyte bone scan and without gross intraoperative signs of infected knee prosthesis. The finding should be carefully evaluated for contamination. If high suspicion for masked infection exists, six weeks of parenteral antibiotic should be administered.

\section{Outcome of treatment with surgical revision}

As a rule, revision Total Knee Arthroplasty offers inferior results and higher complication rates compared to primary arthroplasty [33].

According to the published data the successful functional results following the treatment of late infection of a total knee arthroplasty by a two-stage re-implantation of a new prosthesis should be expected in about $90 \%$ of patients [34, 35, 36, 37].

In spite of its high personal and financial burden the two-stage re-implantation is recognized as the most reliable method for eradicating infection [38]. Although one-stage revision is appealing and less technically demanding, the risk of re-infection is a deterring factor.

Two-stage revision procedures may encounter bone loss, obscure landmarks, structural weakness and soft tissue deficiency, which may result in continued pain, decreased mobility 
and rarely fractures. Nevertheless, the success rate of this method was found to be in the range of $82-93 \%$, whereas the success rate of the one-stage procedure was of $71-81 \%$ [34]. Therefore two-stage re-implantation technique represents the procedure of choice for definitive eradication of infection and preservation of knee function.

According to the published data on one-stage revisions (Table 1) in the large series of patients, with mid-term follow up, around $80 \%$ success rate in eradication of infection should be expected.

\begin{tabular}{ccccc}
\hline Author & Year & No. of patients & Follow-up duration & Success rate* $^{*}$ \\
\hline Foerster et al ${ }^{39}$ & 1991 & 104 & $5-15$ years & $80 \%$ \\
\hline Lu et al $^{49}$ & 1997 & 8 & 20.1 months & $100 \%$ \\
\hline Siegel et al ${ }^{50}$ & 2000 & 31 & $2-15$ years & $71 \%(22 / 31)$ \\
\hline Buechel et al ${ }^{27}$ & 2004 & 22 & 10.2 years & $90.9 \%$ \\
\hline Soudry et al42 & 2009 & 20 & $80 \%(16 / 20)$ \\
\hline Rates of infection eradication & & & & \\
\hline
\end{tabular}

Table 1. Results of one-stage knee revision arthroplasty

As early as 1983, Windsor and Insall reported a success rate of $97.4 \%$ in two-stage revision surgery in 38 patients, with four years of follow-up, but other reports had slightly lower success rates, around 90\% (Table 2).

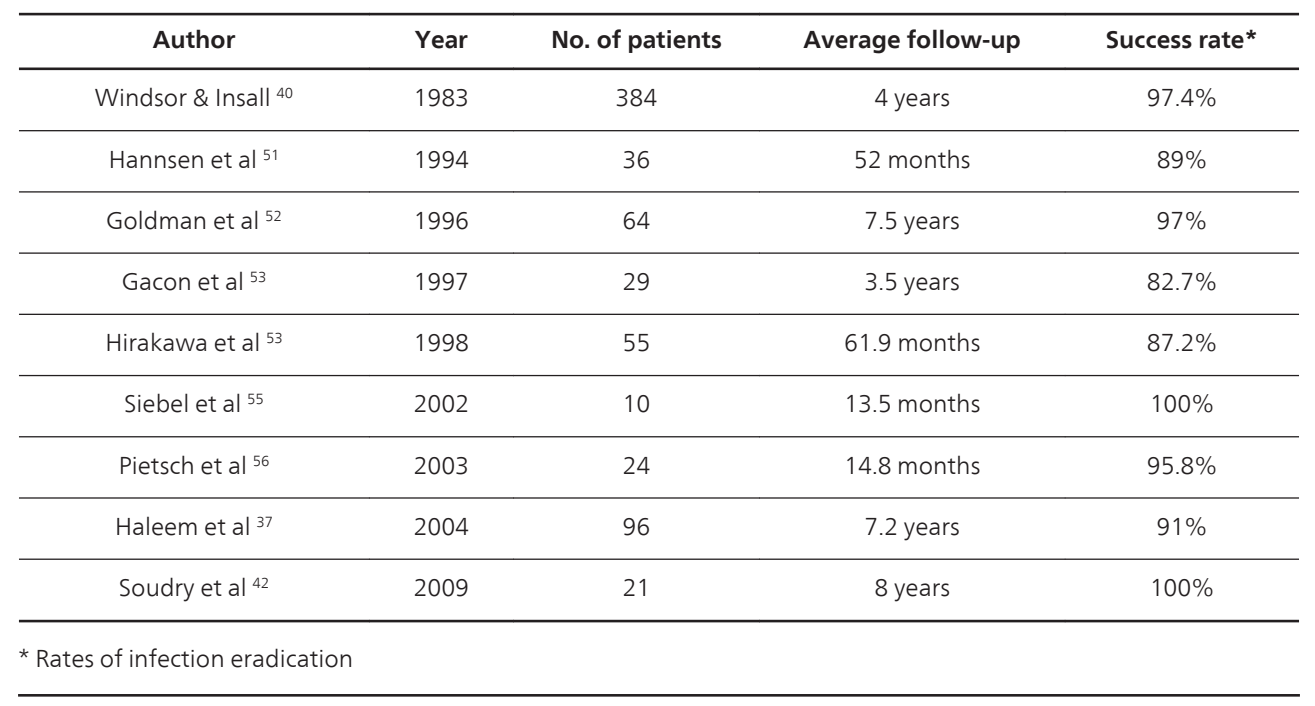

Table 2. Results of two-stage knee revision arthroplasty 
In our series of 43 patients with infected TKA, with characteristic $50 \%$ rate of infection with Staphylococcal strains [7], twenty patients underwent a one-stage procedure and 21 patients underwent a two-stage procedure. Our overall data indicate $83 \%$ postoperative satisfaction with $87 \%$ good and excellent results after revision [41,42]. After an average follow-up of 8 years, subjective satisfaction was reported by $80 \%$ of patients without any evidence of reinfection in the whole group of these patients. However in one-stage group a recurrent infection was noted in $20 \%$ of cases. We use a constrained design of revision prosthesis in order to overcome the expected soft tissue insufficiency in the revised knee (Figure 5).
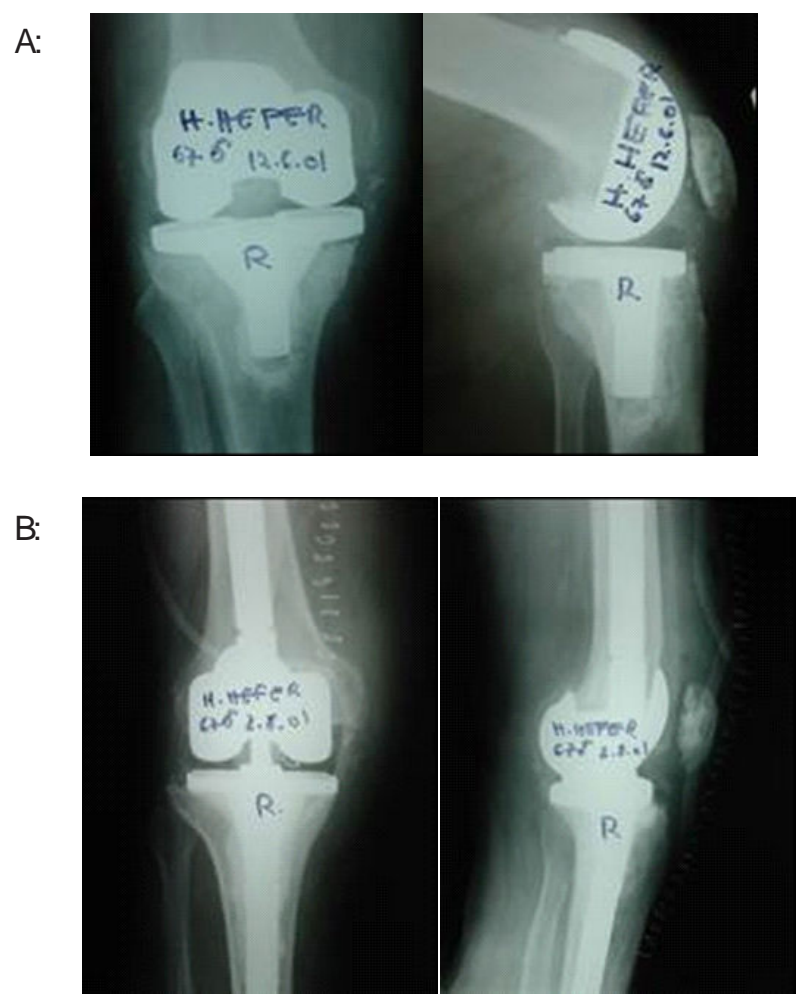

Figure 5. A : Knee radiographs ( Anterior-Posterior and Lateral views). Radiolucency is evident around the tibial component indicating septic loosening. B: Knee radiographs (Anterior-Posterior and Lateral views) following revision with a constrained type prosthesis (CCK).

\section{Salvage surgical procedures}

In failed treatment of revision TKA or in case of a multioperated knee and a debilitated patient another surgical procedure might be required for limb salvage. 


\subsection{Knee arthrodesis}

Knee arthrodesis should be considered as a therapeutic option when other described above techniques have failed, especially in young patients with high functional demands or in patients with extensive deformities, advanced alterations of the extensor mechanism, deficient soft tissues, immunosuppression or infections by highly virulent bacteria. Arthrodesis provides a stable and pain-free limb. However, there is no flexion and the function of the knee is sacrificed, causing an advanced functional impairment. This is generally an irreversible situation. The procedure can be performed with intramedullary nail, metallic plate or external fixation [43, 44] (Figure 6). We have a good clinical experience using the Ilizarov external fixator for this purpose. We used this method in twelve consecutive patients following failed revision TKA surgery performed as treatment for infected initial knee prosthesis. Solid fusion was achieved in all patients within an average healing time of 27.6 weeks. Average shortening of the affected lib was $3.7 \mathrm{~cm}$. We concluded that the Ilizarov fixator for knee arthrodesis after failed TKR produced favorable results and should be considered for the use by surgeons who are familiar with this technique [44]. The success is dependent on the proficiency of the surgeons in Ilizarov method and patient cooperation.

A:

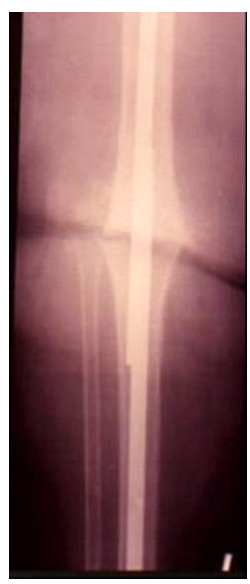

B:

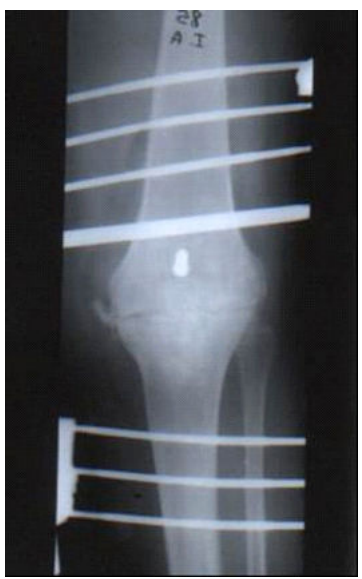

C.

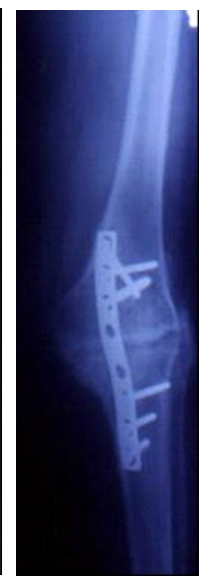

D:

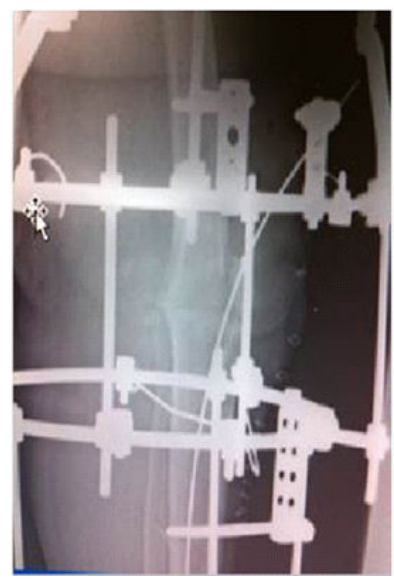

Figure 6. Radiographs of fused knees, following failed revision of TKA, by: A: Intramedullary nail, B: Tubular external fixator. C: Internal fixation by plate and screws, D: Ilizarov external fixator.

\subsection{Resection arthroplasty}

By this salvage method a permanent removal of the implant and cement with local debridement, without re-implantation, are performed. The purpose of this technique is to create a false joint that may allow a certain range of motion. The leg is immobilized for a period between 3 and 6 months in order to allow the soft tissues retraction with creation of free area 
for movement with a certain degree of stability. Candidates for this type of treatment are patients with low functional demands [45].

\subsection{Limb amputation}

This technique should be considered the last resort when dealing with salvage of a prosthetic infection. Its indications are as follows: an uncontrolled infection that threatens the patient's life, large bone loss and severe soft tissue defects [46]. Functional results tend to be extremely poor and patients often end up in a wheelchair. However a successful above knee amputation may provide the best function for patients who otherwise would have a functionless knee joint. In the past limb amputation was required most frequently in infected TKA with cemented stem hinges.

\section{Future: Prosthetic design "tuned" to prevention of periprosthetic infection}

The best solution is to prevent infection rather than treat it. Nowadays the trend is to design an implant that is less susceptible to infection by using surfaces that will be resistant to bacterial adhesion and generation of biofilm. These designs will be appropriate to prevent infection originating via hematogeous spread. Another approach is to use local slowly released antibacterial agents, such as antibiotics or chemical free radicals, that will keep an efficient periprosthetic high concentration antimicrobial milieu in order to prevent biofilm bacterial masking [47]. This is a very important factor since the effective concentration of antibiotics for penetration of biofilm masking should be 1000 times higher than can be achieved following they usual oral or parenteral administration.

Most of the efforts for generation of anti-biofilm surfaces of the prostheses are still in development stage and still have not gain wide clinical use. Currently three main directions are utilized for this purpose. The most common method is to use titanium surfaces that release bactericidal superoxide radicals [48]. This method is especially appealing since $\mathrm{TiO}_{2}$ is has no significant cytotoxic effect on mammalian cells. We observed that human osteoblast-like cells in culture remain viable after exposure to high concentration of $\mathrm{TiO}_{2} 0.1 \mathrm{~mm}$ granules in culture media $(10 \% \mathrm{v}: \mathrm{v})$. Another metal that has bactericidal properties is silver. There are a lot of efforts in designing prosthetic surfaces containing silver [48]. We found that it has a bactericidal effect on different Staphylococci strains, but Pseudomonas aeruginosa remained resistant to its high concentration $(10 \% \mathrm{v}: \mathrm{v})$. The main problem with the use of silver for prosthetic coating is its toxicity to the host cells. We observed a profound cytotoxic effect in cultures of human osteoblast-like cells exposed to $0.1 \mathrm{~mm}$ granules of silver in culture media in bactericidal concentration. For this reason the surfaces coated by $\mathrm{TiO}_{2}$ have a better bactericidal potential for clinical use.

There is also a possibility to use immobilized antibiotic coverage for prosthetic surfaces. This method is still has not reached a proved clinical use [48]. 
Currently the widespread method of prosthetic fixation with methyl methacrylate bone cement, containing broad spectrum antibiotics, is the only proven way to create an antimicrobial periprosthetic surrounding. The uncontrolled release of the antibiotics and potential reduced fixation characteristics of the cement containing antibiotics are the main disadvantage of this method, but it is no clinical evidence that might support these concerns.

\section{Conclusion}

Despite considerable advances in surgical techniques and preoperative care, a $0.5-2 \%$ prevalence of infection in total knee arthroplasty (TKA) still poses a great challenge in the treatment of this devastating and costly complication. Current solutions to treat periprosthetic infection remain imperfect. Treatment strategy varies from conservative lifelong antibiotic suppression therapy in the very high risk patient, arthroscopic or surgical debridement, revision in one or two-stage, arthrodesis or resection arthroplasty as a salvage procedure, and amputation in life-threatening conditions. The decision on the best method of treatment should be personalized to the patient's general health, the severity of the infection and the complexity of the surgery. Currently most of the surgeons have adopted the two-stage protocol, where prosthetic removal, debridement and culture-specific I.V. therapy prior to re-implantation are regarded as standards of care. Although one-stage revision procedure is practiced by some, there is no clear evidence to define when this procedure can be safely applied, because there is no sufficient reliable data on a clinical reliability of this approach. The quest to perform one-stage revision should be continued, as two-stage operations classify the patient in a multiple operations category, with all the resulting potential complications, such as arthrodesis and amputation. Nevertheless, the threat of re-infection after the one-stage procedure surpasses the potential benefits. Judicious selection of patients is the key for successful mode of treatment. Currently the two-stage exchange arthroplasty, with all its inherent problems and drawbacks, allows only a partial success in treatment of TKA infection. New modalities or avenues for treatment of prosthetic infection are desirable.

\section{Author details}

Michael Soudry ${ }^{1 *}$, Arnan Greental ${ }^{2}$, Gabriel Nierenberg ${ }^{2}$, Mazen Falah² and Nahum Rosenberg ${ }^{2}$

*Address all correspondence to: michael.soudry@gmail.com

1 Department of Orthopaedic Surgery, Hillel Yaffe Medical Center, Hadera, Israel

2 Rambam Health Care Campus, Dept of Orthopaedic Surgery. Haifa, Israel 


\section{References}

[1] Zimmerli W., Trampuz A., Ochsner PE. Prosthetic-joint infections. N Engl J Med 2004;351(16):1645-54.

[2] Kurtz SM., Ong KL., Lau E., Bozic KJ., Berry D., Parvizi J. Prosthetic joint infection risk after TKA in the Medicare population. Clin Orthop Relate Res 2010: 468(1):52-56.

[3] Bengtson S., Knutson K. The infected knee arthroplasty. A 6-year follow-up of 357 cases. Acta Orthop Scand 1991; 62(4): 301-11.

[4] Hansen AD., Rand JA. Evaluation and treatment of the infection at the site of a total hip and knee arthroplasry. Instr Course Lect 1999;48:111-122.

[5] Ellenrieder et al. Two-stage revision of implant-associated infections after total hip and knee arthroplasty. GMS Krankenhaushygiene Interdisziplinar 2011;6(1):1-8.

[6] Chiang ER et al. Comparison of articulating and static spacers regarding infection with resistant organisms in total knee arthroplasty .Acta Orthopaedica 2011; 82(4): 460-64.

[7] Peel TN., Buising KL., Choong PFM. Prosthetic joint infection: challenges of diagnosis and treatment. ANZ J Surg; $81: 32-39$.

[8] Fey PD., Olson ME. Current concepts in biofilm formation of Staphylococcus epidermidis. Future Microbiol 2010 5(6): 917-933.

[9] Sendi P., Zimmerli W. Challenges in periprosthetic knee-joint infection. Int J Artif Organs 2011; 34(9):947-956.

[10] Schafroth M., Zimmerli W., Brunazzi M., Ochsner PE. Infections. In: Ochsner PE ed. Total hip replacement. Berlin: Springer Verlag; 2003. p65-90.

[11] Zimmerli W., Trampuz A., Ochsner PE. Prosthetic-joint infections. N Engl J Med 2004;351(16):1645-54.

[12] Giuleri SG., Graber P., Ochsner PE., Zimmerli W. Management of infection associated with THA according to a treatment algorithm. Infection; 2004; 32(4):222-8.

[13] Smith SL., Wastle ML., Forster I. Radiolonuclide bone scintigraphy in the detection of significant complications after total knee joint replacement. Clin Radiol; 2001: 56: 221-4.

[14] Hain SF., O’Doherty MJ., Smith MA. Functional imaging and the orthopedic surgeon. J. Bone Joint Surg Br. 2002; 84:315-21.

[15] Shih LY., Wu JJ., Yang DJ. Erythrocyte sedimentation rate and CRP values with THA. Clinic Orthop 1987:225:238-46. 
[16] Di Cesare PE., Chang E., Preston CF., Liu CJ. Serum Interleukin-6 as a Marker of Periprosthetic Infection Following Total Hip and Knee Arthroplasty. J Bone Joint Surg Am 2005; 87(9):1921-1927.

[17] Marian BD., Martin DS., Levine MJ., Booth RE., Tuan RS. Polymerase Chain. Reaction Detection of Bacterial Infection in Total Knee Arthroplasty. Clinical Orthop 1996; 331: 11-22.

[18] Trampuz A., Piper K., Jacobson M. et al. Sonication of removed hip and knee prostheses for diagnosis of infection. N Engl J Med 2007;357:654-63.

[19] Tampuz A., Hansen AD., Osmon DR., Mandrekar J., Steckekberg JM., Patel R. Advances in the laboratory diagnosis of prosthetic joint infection. Rev Med Microbiol $2003 ; 14: 1-14$.

[20] Parvizi J., Della Valle CJ. In: AAOS Clinical Practice Guideline. Diagnosis and Treatment of periprosthetic joint infections of the hip and knee. J. Am Acad Orthop Surg 2010;18:771-772.

[21] Grogan TJ., Dorey F., Rollins J. et al. Deep sepsis following TKA. JBJS A, 1986: 68:226-234.

[22] Johnson DP., Bannister GC. The outcome of infected arthoplasty of the knee. JBJS B 1986:68;289-291.

[23] Do CSO., Beauchamp CP., Clarke HD., Spangehl MJ. A Two-stage Retention Debridement Protocol for Acute Periprosthetic joint infection. Clin Orthop Relat Res. 2010: Aug;468(8):2029-38.

[24] Parvizi J., Ghanem E., Sharkey P. Prosthetic Joint Infections. Clin Orthop Relat Res 2007; 461:44-47.

[25] Coventry MB., Beckenbaugh RD., Nolan DR., Ilstrup DM. 2,012 total hip arthroplasties. A study of postoperative course and early complications. J Bone Joint Surg Am. 1974;56(2):273-84.

[26] Parkinson RW., Kay PR., Rawal A. A case for one-stage revision in infected totalknee arthroplasty. Knee 2011; 18:1-4.

[27] Buechel FF. The Infected Total Knee Arthroplasty. Just When You Thought It Was Over. The Journal of Arthroplasty 2004; Suppl 4: 19.

[28] Fink B., Gebhard A., Fuerst M., Berger I., Schafer P. High Diagnostic Value of Synovial Biopsy in Periprosthetic Joint Infection of the Hip. Clin Orthop Relat Res. in press.

[29] Nickinson RS.J, Board TN., Gambhir AK., Porter ML., Kay PR. Two stage revision knee arthroplasty for infection with massive bone loss. A technique to achieve spacer stability. Knee 2012;19: 24-27. 
[30] Kohl S., Evangelopoulos DS., Kohlhof H., Krueger A., Hartel M., Roeder C., Eggli S. An intraoperatively moulded PMMA prostheses-like spacer for two-stage revision of infected total knee arthroplasty. Knee 2011;18: 464-469.

[31] Choi HR., Malchau H., Bedair H. Are Prosthetic Spacers Safe to Use in 2-Stage Treatment for Infected Total Knee Arthroplasty? J of Arthroplasty 2012; in press.

[32] Macmull S., Bartlett W., Miles GW., Blunn J., Pollock RC., Carrington RWJ., Skinner JA., Cannon SR., Briggs TWR. Custom-made hinged spacers in revision knee surgery for patients with infection, bone loss and instability. Knee 2010;17: 403-406.

[33] Joseph TN., Chen AL., Di Cesare PE. Use of antibiotic-impregnated cement in total joint arthroplasty. J Am Acad Orthop Surg 2003;11:38-47.

[34] Hanssen AD., Rand JA. Evaluation and treatment of infection at the site of a total hip or knee arthroplasty. J Bone Joint Surg 1998; 80:910-922.

[35] Hoad-Reddick DA., Evans CR., Norman .P, Stockly I. Is there a role for extended antibiotic therapy in a two-stage revision of the infected knee arthroplasty?. J Bone Joint Surg Br 2005; 87-B:171-174.

[36] Freeman MA., Sudlow RA., Casewell MW., Radcliff SS. The management of infected total knee replacements. J Bone Joint Surg Br 1985; 67:764-768.

[37] Haleem AA., Berry DJ., Hanssen AD. Mid-term to long-term follow-up of two-stage reimplantation for infected total knee arthroplasty. Clin Orthop. 2004: 428:35-39.

[38] Wilde AH., Ruth JT. Two-stage reimplantation in infected total knee arhroplasty. Clin Orthop 1988; 236:23-35.

[39] Von Foerster G., Klüber D., Käbler U. Mid- to long-term results after treatment of 118 cases of periprosthetic infections after knee joint replacement using one-stage exchange surgery]. Orthopade. 1991 Jun;20(3):244-52.

[40] Windsor R., Insall J. Management of the infected total knee arthroplasty. In: InsallScott, eds. Surgery of the Knee. 1st ed. Churchill Livingstone, New York, 1983. 959-974.

[41] Soudry M., Greental A., Niereberg G., Falah M. One and two-stage revision in infected TKA. JBJS B. 2005: Vol 87-B. Supp III: 389.

[42] Soudry M., Nierenberg G., Msika C., Jontschew DA., Falah M. One vs two-stage revision of infected knee arthroplasty. Portugese Journal of Orthopaedics. 2009: Vol 17 (4): $1-11$.

[43] Klinger HM., Spahn G., Schultz W., Baums MH. Arthrodesis of the knee after failed infected total knee arthroplasty. Knee Surg Sports Traumatol Arthrosc.2006; 14(5): 447-53.

[44] David R., Shtarker H., Horesh Z., Tsur A., Soudry M. Knee Arthrodesis with the Ilizarov after failed knee arthroplasty. Orthopedics, 2001 Jan: 24(1):33-6. 
[45] Falahee MH., Matthews LS., Kaufer H. Resection Arthroplasty as a salvage procedure for a knee with infection after TKA. JBJS A 1987: 69: 1013-1021.

[46] Pring DJ., Marks, Angel JC. Mobility after amputation for failed knee replacement. JBJS B 1988: 70: 770-771).

[47] Vasilev K., Cook .J, Griesser HJ. Antibacterial surfaces for biomedical devices. Expert Rev Med Devices 2009; 6(5): 553-567.

[48] Visai L., De Nardo L., Punta C., Melone L., Cigada A., Imbriani M., Arciala CR. Titanium oxide antibacterial surfaces in biomedical devices. Int J Artif Organs 2011; 34(9):929-946.

[49] Lu H., Kou B., Lin J. One-stage reimplantation for the salvage of total knee arthroplasty complicated by infection. Zhonghua Wai Ke Za Zhi. 1997 Aug;35(8):456-8.

[50] Siegel A., Frommelt L., Runde W. Therapy of bacterial knee joint infection by radical synovectomy and implantation of a cemented stabilized knee joint endoprosthesis. Chirurg. 2000 Nov;71(11):1385-91.

[51] Hanssen AD., Rand JA., Osmon DR. Treatment of the infected total knee arthroplasty with insertion of another prosthesis. The effect of antibiotic-impregnated bone cement. Clin Orthop Relat Res. 1994 Dec;(309):44-55.

[52] Goldman RT., Scuderi GR., Insall JN. 2-stage reimplantation for infected total knee replacement. Clin Orthop Relat Res. 1996 Oct;(331):118-24.

[53] Gacon G., Laurencon M., Van de Velde D., Giudicelli DP. Two stages reimplantation for infection after knee arthroplasty. Apropos of a series of 29 cases. Rev Chir Orthop Reparatrice Appar Mot. 1997;83(4):313-23.

[54] Hirakawa K., Stulberg BN., Wilde AH., Bauer TW., Secic M. Results of two-stage reimplantation for infected total knee arthroplasty. J Arthroplasty. 1998 Jan;13(1):22-8.

[55] Siebel T., Kelm J., Porsch M., Regitz T., Neumann WH. Two-stage exchange of infected knee arthroplasty with a prosthesis-like interim cement spacer. Acta Orthop Belg. 2002 Apr;68(2):150-6.

[56] Pietsch M., Wenisch C., Traussnig S., Trnoska R., Hofmann S. Temporary articulating spacer with antibiotic-impregnated cement for an infected knee endoprosthesis. Orthopade. 2003 Jun;32(6):490-7. 